Pacific Journal of Mathematics

COMPLEMENTATION IN THE LATTICE OF CONVERGENCE
STRUCTURES 


\title{
COMPLEMENTATION IN THE LATTICE OF CONVERGENCE STRUCTURES
}

\author{
C. V. RIECKE
}

This paper classifies the various complements in the lattice of convergence structures and considers some of the properties shared by given structures and their relative complements or differences.

1. Introduction. The lattice of all convergence structures on a nonempty set has been investigated by several authors with some consideration given to the smaller lattices of pseudotopologies and pretopologies. The purpose here will be to show the lattice of convergence structures is a Stone lattice and has pseudo-differences, completely characterize relative pseudo-complements and pseudodifferences, establish that some types of convergence structures retain their classification after finding relative complements or differences and exhibit certain standard lattice operators as homomorphisms.

Lattice definitions follow those of Rasiowa and Sikorski [11], Birkhoff [2], and Szasz [15]. An element $x$ of a lattice $L$ is compact if whenever $x \leqq \vee\left\{y_{j} \mid j \in J\right\}$ implies that $x \leqq \vee\left\{y_{j} \mid j \in K\right\}$ for some finite subset $K$ of $J$. The lattice $L$ is compactly generated if each element of $L$ is the join of compact elements. Cocompact elements and cocompactly generated lattices are defined dually. An element $z$ in $L$ is the pseudo-complement of $x$ relative to $y(x * y)$ if $z$ is the greatest element such that $x \wedge z \leqq y$. If $L$ has a least element 0 , the pseudo-complement $-x$ of $x$ is the greatest element for which $x \wedge(-x)=0$. A Brouwerian lattice is one in which the relative pseudo-complement of any two members always exists; a Brouwerian lattice with a least element is pseudo-Boolean and a pseudo-Boolean lattice is a Stone lattice if there exists a greatest element 1 and $(-x) \vee(--x)=1$ for every $x$ in $L$. An element $z$ is the pseudo-difference of $y$ and $x(y-x)$ if $z$ is the least element such that $y \leqq x \vee z$.

Definitions on convergence structures used herein can be found in [1], [7] or [9]. $C(X)$ will denote the family of all convergence structures on a nondegenerate set $X, F(X)$ the lattice of filters on $X$ and $U(X)$ the set of ultrafilters. In this paper the power set or improper filter is considered a member of $F(X)$. This makes $F(X)$ a complete lattice and facilitates the description of pseudo-differences in terms of the filter quotients of Ward [16].

For a point $x, \dot{x}$ will be the principal filter on $X$ generated by $\{x\}$ while in $F(X)[\mathscr{F}]$ signifies the principal filter generated by a 
filter $\mathscr{F}$ on $X$ and $(\mathscr{F} x)=\{\mathscr{G} \mid \mathscr{G} \cap \dot{x} \subset \mathscr{F}\}$. For filters $\mathscr{F}$ and $\mathscr{G}$ on $X, \mathscr{F}+\mathscr{G}$ is the filter sum or the lattice join in $F(X)$ (see [14]).

For convenience the terms pseudotopology, pretopology and topology are abbreviated ps., pre. and top. The usage of these terms is that described by Fischer [6] and Choquet [5].

\section{Lattice structure of $C(X)$.}

DEFINITION 2.1. A convergence structure on a nonempty set $X$ is a map $q: X \rightarrow \beta(F(X))$ such that for each $x \in X, \dot{x} \in q(x)$ and if $\mathscr{F} \in q(x), \mathscr{F} \cap \dot{x} \in q(x)$ and $\mathscr{G} \in q(x)$ for all filters $\mathscr{G}$ finer than $\mathscr{F}$.

A filter $\mathscr{F}$ is said to $q$-converge to $x$ if $\mathscr{F} \in q(x)$. On $C(X)$, define a partial order by $q \leqq r$ if and only if $r(x) \leqq q(x)$ for all $x \in X$. Then $C(X)$ with this order becomes a complete, distributive lattice $[7,8]$ in which for any $\left\{q_{j} \mid j \in J\right\}$, a subset of $C(X),\left(\vee q_{j}\right)(x)=$ $\cap q_{j}(x)(j \in J)$ and $\left(\wedge q_{j}\right)(x)=\cup q_{j}(x)(j \in J)$.

One can observe that the lattice of convergence structures contains a least and a greatest element as well as atoms and coatoms. The greatest element is the discrete topology $D$ and the least element is the indiscrete topology $I$. A convergence structure $q$ is an atom in $C(X)$ if, for some $x \in X, q(x)=F(X) \backslash\{\{X, X \backslash\{x\}\}\}$ and $q(y)=F(X)$ for $y \neq x$. Coatoms are of the form $(x, \mathscr{F})$ for some $x \in X$ and $\mathscr{F} \in U(X)$ where $(x, \mathscr{F})$ is the finest convergence structure $q$ (in fact, topology) for which $\mathscr{F} \in q(x)$. While each member of $C(X)$ is preceded (succeeded) by an atom (coatom) the lattice $C(X)$ is not atomic (not coatomic). The sublattice generated by the atoms of $C(X)$ is isomorphic to the power set of $X$ whereas the coatoms generate a sublattice isomorphic to the lattice of pseudotopologies [4].

LEMMA 2.2. A convergence structure $q$ is a compact element of $C(X)$ if and only if there exists a finite subset $Y=\left\{x_{1}, \cdots, x_{n}\right\}$ of $X$ and for each $x_{k}$ a finite subset $\{\mathscr{F}(j, k) \mid j=1, \cdots, n(k)\}$ of $F(X)$ such that $q(x)=F(X)$ if $x \notin Y$ and $q\left(x_{k}\right)=F(X) \backslash \cup\left(F(j, k) x_{k}\right)(j=1$, $\cdots, n(k))$.

Proof. If $A=\{x \in X \mid q(x) \neq F(X)\}$ is infinite, for each $x$ in $A$ define $q_{x}$ in $C(X)$ to be $q_{x}(y)=F(X)$ if $y \neq x$ and $q_{x}(x)=q(x)$. Then $q=\vee q_{x}(x \in A)$ but $q \neq \vee q_{x}(x \in B)$ for any finite subset $B$ of $A$ and $q$ is not a compact lattice element.

Suppose for some $x \in A$ no finite family $\mathscr{F}_{1}, \cdots, \mathscr{F}_{m}$ of filters on $X$ exists with $q(x)=F(X) \backslash \cup\left(\mathscr{F}_{j} x\right) \quad(j=1, \cdots, m)$. For each $\mathscr{F} \in F(X)$ with $\mathscr{F} \notin q(x)$, define $q(\mathscr{F})$ in $C(X)$ by $q(\mathscr{F})(y)=q(y)$ 
for $y \neq x$ and $q(\mathscr{F})(x)=F(X) \backslash(\mathscr{F} x)$. Now $q=\vee q(\mathscr{F})(\mathscr{F} \in q(x))$ but $q$ is not the join of any finite subset so again $q$ could not be compact. The converse follows readily.

THEOREM 2.3. $C(X)$ is compactly generated.

Proof. If $q \in C(X)$, for every $x \in X$ and filter $\mathscr{F}$ not in $q(x)$, let $q(x, \mathscr{F})$ in $C(X)$ be $q(x, \mathscr{F})(y)=F(X)$ if $y \neq x$ and $q(x, \mathscr{F})(x)=$ $F(X) \backslash(\mathscr{F} x)$. Each $q(x, \mathscr{F})$ is compact by Lemma 2.2 and $q=$ $\vee q(x, \mathscr{F})(x \in X, \mathscr{F} \notin q(x))$.

LEMma 2.4. A convergence structure $q$ is a cocompact element of $C(X)$ if and only if $\{x \mid q(x) \neq[\dot{x}]\}$ is finite and if $q(x) \neq[\dot{x}]$ there exists a finite subset $\left\{\mathscr{F}_{1}, \cdots, \mathscr{F}_{n}\right\}$ of $F(X)$ such that $q(x)=$ $\left\{\mathscr{F} \in F(X) \mid \mathscr{F}_{j} \subseteq \mathscr{F}\right.$ for some $\left.j=1, \cdots, n\right\}$.

Proof. The proof parallels the procedure in Lemma 2.2.

One can note from Lemma 2.4 that each cocompact member of $C(X)$ is the meet of a finite set of topologies of the form $(x, \mathscr{F})$ for $\mathscr{F}$ a filter on $X$. One immediate consequence follows from Lemma 2.4.

THEOREM 2.5. $C(X)$ is cocompactly generated.

Proof. If $q \in C(X), q=\wedge\{(x, \mathscr{F}) \mid \mathscr{F} \in q(x), x \in X\}$ and each $(x, \mathscr{F})$ is cocompact by Lemma 2.4 .

If $L$ is a lattice, $x \in L, A \subset L$ and $\mathscr{F}$ is a filter on $L, A^{-}=$ $\{y \mid x \leqq y$, all $x$ in $A\}$ and $\mathscr{F}^{-}=\cup\left\{A^{-} \mid A \in \mathscr{F}\right\}$. The corresponding lower bounds are designated by $A^{+}$and $\mathscr{F}^{+} . A$ filter $\mathscr{F}$ order converges to $x$ if $x=\vee \mathscr{F}^{+}=\wedge \mathscr{F}^{-}$. The order topology on $L$ is defined as that topology whose closed sets are those sets $A$ which contain all points to which filters that contain $A$ converge. Kent and Atherton [10] have shown that in a compactly and cocompactly generated lattice (i.e., bicompactly generated) order convergence is topological. Hence Theorems 2.3 and 2.5 with the result of Kent and Atherton imply that order convergence in the lattice of convergence structures is topological.

An inspection of the lattice operations will quickly show that $C(X)$ is not a Boolean lattice. It is possible, however, to show that $C(X)$ has pseudo-complements, relative pseudo-complements and pseudo-differences as well as present an internal characterization of each. 
THEOREM 2.6. $C(X)$ is Brouwerian.

Proof. If $q$ and $r$ are in $C(X)$, define $q * r: X \rightarrow \beta(F(X))$ by $q * r(x)=\{\mathscr{F} \mid \mathscr{F} \supseteqq \dot{x}$ or $\mathscr{G} \cap \dot{x} \subseteq \mathscr{F}$ for some $\mathscr{G} \in r(x) \backslash q(x)\}$. The map $q * r$ is a convergence structure and is the pseudo-complement of $q$ relative to $r$ in $C(X)$.

Corollary 2.7. $C(X)$ is a Stone lattice.

Proof. In any Brouwerian lattice with least element $I$, the pseudo-complement of $q$ is $-q=q * I$. In $C(X)$ this means that if $A=\{x \in X \mid q(x) \neq F(X)\},-q(x)=[\dot{x}]$ if $x \notin A,-q(x)=F(X)$ if $x \in A$ and $(--q) \vee(-q)=D$.

The characterization of the pseudo-complements of members of the lattice of convergence structures gives rise to a natural map between the class of all regular elements of $C(X)$ and $\beta(X)$. The subsequent corollary has appeared in [12].

COROLlary 2.8. The maximal Boolean sublattice of $C(X)$ is isomorphic to $\beta(X)$.

Pseudo-differences were used in [12] to exhibit a classification of the group of lattice automorphisms of $C(X)$. Pseudo-differences in $C(X)$ are closely related to the concept of a quotient filter defined by Ward [16]. The properties of pseudo-differences will be generalized slightly to an arbitrary distributive lattice.

LEMma 2.9. In a distributive lattice $L$, for any cofinal subsets $A$ and $B$, let $A: B=\{x \mid x \vee y \in A$ for all $y \in B\}$. Then:

(i) $A: B$ is cofinal.

(ii) If $A$ is a filter, $A$ : $B$ is a filter.

(iii) If $C$ is a cofinal subset of $L$ with $B \cup C \subseteq A$, then $C \leqq A: B$.

(iv) $A \subseteq A: B$.

(v) $(A: B) \cap B \subseteq A$.

Proof. The proof parallels a proof of a similar theorem for power set lattices by Schmidt [14].

Definition 2.10. Tne set $G: H$ in Lemma 2.9 will be called the quotient set of $G$ and $H$.

THEOREM 2.11. $C(X)$ is pseudo-differenced. 
Proof. For $q$ and $r$ in $C(X)$, define $q-r: X \rightarrow \beta(F(X))$ by $(q-r)(x)=q(x): r(x)$. That $q-r$ is a convergence structure on $X$ follows from (i) of Lemma 2.9 and the fact that if $\mathscr{F} \in(q-r)(x)$ and $\mathscr{G} \in r(x),(\dot{x} \cap \mathscr{F})+\mathscr{G}=(\dot{x}+\mathscr{G}) \cap(\mathscr{F}+\mathscr{G})$ where $\mathscr{F}+\mathscr{G}$ is the filter generated by $\mathscr{F}$ and $\mathscr{G}$ as used in [14].

Part (v) of Lemma 2.9 implies $r(x) \cap(q-r)(x)=r(x) \cap(q(x)$ : $r(x)) \subseteq q(x)$ for each $x$ in $X$ and $q \leqq r \vee(q-r)$. If $s \in C(X)$ with $q \leqq r \vee s$, then $r(x) \cap s(x) \subseteq q(x)$ and part (iii) of Lemma 2.9 implies $s(x) \subseteq q(x): r(x)$ so $q-r \leqq s$ and $q-r$ is the pseudo-difference of $q$ and $r$.

3. Relative pseudo-complements in $C(X)$.

Definition 3.1. A subset $A$ of a lattice $L$ is prime with respect to joins in $L$ if $x \vee y \in A$ implies $x \in A$ or $y \in A$.

LemMA 3.2. For $q$ in $C(X), \quad q *(r \vee s)=(q * r) \vee(q * s)$ for all convergence structures $r$ and $s$ in $C(X)$ if and only if $q(x) \backslash[\dot{x}]$ is prime with respect to joins in $F(X)$ for every point $x$.

Proof. Since in any Brouwerian lattice $q *(r \vee s) \geqq(q * r) \vee(q * s)$, it suffices to show the condition is equivalent to the reverse inequality.

Assume filters $\mathscr{F}, \mathscr{G}$ exist such that $\mathscr{F}+\mathscr{G} \in q(x) \backslash[\dot{x}]$ and $\mathscr{F}, \mathscr{G} \notin q(x) \backslash[\dot{x}]$. Define $r, s$ in $C(X)$ by $r(y)=s(y)=[\dot{y}]$ if $y \neq x$, $r(x)=[\mathscr{F} \cap \dot{x}]$ and $s(x)=[\mathscr{G} \cap \dot{x}]$. Then $\mathscr{F}+\mathscr{G}$ is in $[(q * r) \vee$ $(q * s)](x)$ but not in $[q *(r \vee s)](x)$ and $(q * r) \vee(q * s) \nsupseteq q *(r \vee s)$.

Conversely, if for some $x \in X$, there exists $\mathscr{F} \notin[q *(r \vee s)](x)$ with $\mathscr{F} \in[(q * r) \vee(q * s)](x)$ then there exist $\mathscr{G} \in r(x) \backslash q(x)$ and $\mathscr{H} \in$ $s(x) \backslash q(x)$ with $\mathscr{G}+\mathscr{H} \subseteq \mathscr{F}$. Now $\mathscr{G}+\mathscr{H} \in r(x) \cap s(x)$ so if $\mathscr{G}+$ $\mathscr{H} \notin q(x), \mathscr{F} \in[q *(r \vee s)](x)$. But $\mathscr{F}, \mathscr{G}, \mathscr{H} \neq \dot{x}$ and $q(x) \backslash[\dot{x}]$ is not prime with respect to joins.

On $C(X)$, define, for any convergence structure $q$, the self-map $f(q)$ by $f(q)(r)=q * r$.

THEOREM 3.3. For any couvergence structure $q$ :

(i) $f(q)$ is a meet-homomorphism

(ii) $f(q)$ is a join-homomorphism and an interior operator iff each $q(x) \backslash[\dot{x}]$ is prime with respect to joins in $F(X)$.

Proof. Part (i) follows from ordinary properties of relative pseudo-complements and (ii) is an immediate consequence of Lemma 3.2 . 
A natural question to consider is whether the relative pseudocomplement of two convergence structures preserves any of the properties of the original structures. Since $q * r$ is the discrete structure whenever $q \leqq r$ such a question appears not to hold much promise of fruitful investigation. One interesting result holds when $q$ is a limitierung (resp. ps., pre., top.).

THEOREM 3.4. If $q$ and $r$ are convergence structures on $X$ with $r$ a limitierung (respectively ps., pre., top.) then $q * r$ is also a limitierung (resp. ps., pre., top.).

Proof. A proof will be exhibited for the case of $r$ a topology. Since $r \leqq q * r$, each $r$ - open set is $q * r$ - open and the $q * r$ - neighborhood filter $N_{*}$ has a base of $q * r$ - open sets for each $x \in X$ with $N_{r}(x) \notin q(x)$ since for these points $q * r(x)=\left[N_{r}(x)\right]$. If $N_{r}(x) \in$ $q(x), q * r(x)$ is discrete and $\{x\}$ is $q * r$ - open so $q * r$ is a topology.

THEOREM 3.5. If $r$ is a $T_{1}\left(T_{2}\right)$ convergence structure, then $q * r$ is $T_{1}\left(T_{2}\right)$ for any convergence structure $q$.

COROLlaRY 3.6. The lattice $C_{1}(X)$ of $T_{1}$ convergence structures on $X$ is Brouwerian.

Proof. Since $C_{1}(X)$ is a principal filter in $C(X)$, the relative pseudo-complements of two $T_{1}$-structures must be the same for both lattices.

4. Pseudo-difference. Since properties of pseudo-differences are perhaps less familiar some of these properties are included for reference.

THEOREM 4.1. If $L$ is a distributive lattice having pseudodifference and a least element, then for any $x, y, z$ in $L$,

(i ) $x-y \leqq x$.

(ii) $x-z \leqq y-z$ if $x \leqq y$.

(iii) $(x-y)-y=x-y$.

If $L$ is complete and completely distributive,

(iv) $\left(\vee x_{j}\right)-y=\vee\left(x_{j}-y\right)(j \in J)$.

(v) $\left(\wedge x_{j}\right)-y \leqq \wedge\left(x_{j}-y\right)(j \in J)$.

Although equality in part (v) of Theorem 4.1 does not always hold even for finite cases, equality can be determined for the lattice of convergence structures.

THEOREM 4.2. For any convergence structure $q$ on $X$, in $C(X)$ : 
(i) $D-q=D$ iff each ultrafilter $q$ - converges to every point.

(ii) $(r-q) \wedge(s-q)=(r \wedge s)-q$ for all $r$, s in $C(X)$ iff $q$ is a limitierung.

Proof. The proof of (i) is direct. A proof of only (ii) will be given.

Assume $q$ is a limitierung, $x \in X$ and $\mathscr{F} \notin(r-q)(x) \cup(s-q)(x)$. Then there exists $\mathscr{G}$, $\mathscr{H}$ in $q(x)$ with $\mathscr{F}+\mathscr{G} \notin r(x)$, $\mathscr{F}+\mathscr{H} \notin$ $s(x)$. Since $\mathscr{G} \cap \mathscr{H} \in q(x)$ and $\mathscr{F}+(\mathscr{G} \cap \mathscr{H}) \leqq(\mathscr{F}+\mathscr{G}) \cap(\mathscr{F}+$ $\mathscr{H}), \mathscr{F}+(\mathscr{G} \cap \mathscr{H}) \notin(r \wedge s)(x)$. This together with Theorem 4.1., part (v) implies the desired equality.

Conversely, if $(r-q) \wedge(s-q) \leqq(r \wedge s)-q$ for all $r, s$ in $C(X)$, suppose $\mathscr{G}_{1}, \mathscr{G}_{2}, \in q(x)$ with $\mathscr{G}_{1}, \mathscr{G}_{2}, \neq \dot{x}$ and $\mathscr{G}_{1} \cap \mathscr{G}_{2} \neq q(x)$. Define $r, s$ in $C(X)$ as follows:

$$
\begin{aligned}
r(x)= & \{\mathscr{F} \in F(X) \mid \mathscr{F} \supseteqq \dot{x} \text { or } \mathscr{G} \subseteq \mathscr{F} \text { for some } \mathscr{G} \in q(x) \\
& \text { with } \left.\mathscr{G} \cap \dot{x} \leqq \mathscr{G}_{1}\right\} \text { and } r(y)=q(y) \text { if } y \neq x, \\
s(x)= & \{\mathscr{F} \in F(X) \mid \mathscr{F} \supseteqq \dot{x} \text { or } \mathscr{G} \cap \dot{x} \subseteq \mathscr{F} \text { for some } \mathscr{G} \in q(x) \backslash \\
& r(x)\} \text { and } s(y)=q(y) \text { if } y \neq x .
\end{aligned}
$$

$\mathscr{G}_{2} \notin r(x)$ since $\mathscr{G}_{1} \cap \mathscr{G}_{2} \notin q(x)$ and similarly $\mathscr{G}_{1} \notin s(x)$. Moreover, $r \wedge s=q$ so $(r \wedge s)-q=I$. Since $\left(\mathscr{G}_{1} \cap \mathscr{G}_{2}\right)+\mathscr{G}_{2} \notin r(x)$ and $\left(\mathscr{G}_{1} \cap\right.$ $\left.\mathscr{G}_{2}\right)+\mathscr{G}_{1} \notin s(x), \quad \mathscr{G}_{1} \cap \mathscr{G}_{2} \notin[(r-q) \wedge(s-q)](x)$, contradicting the assumption and showing $q$ to be a limitierung.

In a fashion similar to that for relative pseudo-complements, a self-map of $C(X)$ can be defined for each convergence structure $q$ by $\bar{f}(q)(r)=r-q$. This pseudo-difference map was used in [12] to determine the group of lattice automorphisms of the lattice of convergence structures where a slightly different definition was used for a convergence structure corresponding to the definition used by Kent [8].

THEOREM 4.3. The pseudo-difference map $\bar{f}(q)$ associated with a given convergence structure $q$ is a lattice homomorphism if and only if $q$ is a limitierung. In addition $\bar{f}(q)$ is an interior operator if and only if $q$ is a limitierung satisfying the condition of (i) of Theorem 4.2.

Proof. The proof is a consequence of Theorem 4.2 and parts (i)-(iv) of Theorem 4.1.

For pseudo-differences the situation regarding pretopologies and topologies is not so clear as for relative pseudo-complements. The next two theorems show some results on preservation of the various 
types of structures.

THEOREM 4.4. If $q$ and $r$ are in $C(X), r-q$ is a limitierung (pseudotopology) if $r$ is a limitierung (pseudotopology).

Proof (for $r$ a pseudotopology). If $\mathscr{F} \in F(X)$ has the property that $\mathscr{G} \in(r-q)(x)$ for every ultrafilter $\mathscr{G}$ with $\mathscr{F} \subseteq \mathscr{G}$ then for any $\mathscr{H} \in q(x)$ and any ultrafilter $\mathscr{K}$ with $\mathscr{F}+\mathscr{H} \subseteq \mathscr{K}, \mathscr{K} \in$ $(r-q)(x)$. But this implies $\mathscr{K} \in r(x), \mathscr{F}+\mathscr{H} \in r(x)$ and $\mathscr{F} \in$ $(r-q)(x)$ so $r-q$ is a pseudotopology.

EXAMPLE 4.5. Let $X$ be an infinite set, $x \in X$ and $\mathscr{F}$ be a free ultrafilter on $X$. For each $y \in X, \dot{y} \in[D-(x, \mathscr{F})](x)$ from which it follows that the filter $\{X\} \in[D-(x, \mathscr{F})](x)$ if $[D-(x, \mathscr{F})]$ is a pretopology. However, $\{X\}+\mathscr{F}=\mathscr{F} \notin D(x)$ so $D-(x, \mathscr{F})$ is not a pretopology even though both original structures are topologies.

LeMmA 4.6. If $q$ and $r$ are in $C(X)$ an ultrafilter $\mathscr{F} q-r$ converges to $x$ if and only if $\mathscr{F} q$-converges to $x$ or does not $r$ - converge to $x$.

THEOREM 4.7. If $q$ and $r$ are convergence structures on $X$ and $(\lambda q)-r$ is a pretopology, then $(\lambda q)-r=\lambda(q-r)$ where $\lambda q$ is the finest pretopology preceding $q$ in $C(X)$.

Proof. Since $\lambda q \leqq q$, by part (ii) of Theorem 4.1, it suffices to show $\lambda(q-r) \leqq(\lambda q)-r$. If $s=(\lambda q)-r$ and $x \in X$, the $s-$ neighborhood filter of $x$ is $N_{s}(x)=\cap\{\mathscr{F} \in U(X) \mid \mathscr{F} \in s(x)\}=\cup\{\mathscr{F} \in$ $U(X) \mid \mathscr{F} \notin r(x)$ or $\mathscr{F} \in \lambda q(x)\}$ by Lemma 4.6. But $N_{q}(x)=\cap\{\mathscr{F} \in$ $U(X) \mid \mathscr{F} \in \lambda q(x)\}=\left\{\cap\{\mathscr{F} \in U(X) \mid \mathscr{F} \in q(x)\}\right.$ which implies $N_{s}(x)=$ $\cap\{\mathscr{F} \in U(X) \mid \mathscr{F} \in(q-r)(x)\}, N_{s}(x) \in \lambda(q-r)(x)$ and $\lambda(q-r) \leqq(\lambda q)-r$.

For $T_{1}\left(T_{2}\right)$ - convergence structures, one can. ascertain precisely the conditions under which the pseudo-difference of two $T_{1}\left(T_{2}\right)$ structures is also $T_{1}\left(T_{2}\right)$ but the conditions are not especially elegant or illuminating. One result on $T_{1}$-structures is worth stating although we omit the proof which can be obtained by a check of the definitions.

THEOREM 4.8. The lattice $C_{1}(X)$ of all $T_{1}$-convergence structures on $X$ has pseudo-differences. In particular, if $q$ and $r$ are $T_{1^{-}}$ structures on $X$, the pseudo-difference $q-r$ in $C_{1}(X)$ is $(q-r)(x)=$ $\{\mathscr{F} \in(q-r)(x) \mid\{x\} \supseteqq \cap A(A \in \mathscr{F})\}$. 
5. Lattice homomorphisms in $C(X)$. The families of limitierungs (resp. pre., ps., top.) are well-known to be complete lattices whose order is the order induced from $C(X)$. Moreover, each of these lattices is a join-complete subsemilattice of $C(X)$. Information on completions of many families of special types of structures in $C(X)$ is contained in [7] or [13]. With each convergence structure $q$ is associated the finest limitierung (pre., ps., or top.) coarser than $q$. This gives rise to maps $\phi, \sigma, \lambda, \omega$ of $C(X)$ into the lattices of limitierungs (ps., pre., top.) where $\phi q(\sigma q, \lambda q, \omega q)$ is the finest limitierung (ps., pre., top.) coarser than $q$. These functions were essentially all defined by Kent [8] or Fischer [6] and subsequent investigations of $\omega q$ and $\lambda q$ appear in $[6,8,9]$. The purpose here is to show that most of these functions are lattice homomorphisms.

THEOREM 5.1.

(i) $\phi(\sigma, \lambda, \omega)$ is a complete meet homomorphism

(ii) $\dot{\phi}$ is a finite join homomorphism

(iii) $\sigma$ and $\lambda$ are complete lattice homomorphisms.

Proof. Most of the appropriate homomorphism properties follow directly from the properties of $C(X)$ and the definition of the maps. Let us show that $\lambda$ is a complete lattice homomorphism. If $\left\{q_{i}\right\}$ is any family of convergence structures on $X, \wedge^{\circ} \lambda q_{i}=\lambda\left(\wedge^{\circ} \lambda q_{i}\right) \leqq$ $\lambda\left(\wedge q_{i}\right)$ by definition of $\lambda\left(\wedge^{\circ}\right.$ in the lattice of pretopologies). If $\mathscr{F} \in \vee^{\circ} \lambda q_{i}(x), N_{i}(x)$, the $q_{i}$-neighborhood filter of $x$, is contained in $\mathscr{F}$ for each $q_{i}$. If $q=\vee q_{i}, N_{q}(x)=\Sigma N_{i}(x) \subseteq \mathscr{F}$ and $\mathscr{F} \in \lambda\left(\vee q_{i}\right)$ (x) so $\vee^{\circ} \lambda q_{i} \geqq \lambda\left(\vee q_{i}\right)$. The necessary reverse inequalities follow directly.

EYAMPLE 5.2. The map $\omega$ is not of necessity join preserving. If $X=\{x, y, z\}$ with $q$ and $r$ defined on $X$ by $r(x)=[\dot{x} \cap \dot{z}], r(y)=$ $[\dot{x} \cap \dot{y}], r(z)=[\dot{y} \cap \dot{z}]$ with $q(x)=[\dot{x} \cap \dot{y}]$ and $q$ discrete at $y$ and $z$, $\omega(q \vee r)=D$ but $\omega q \vee \omega r=\omega q \neq D$.

EXAMPLE 5.3. Let $N$ be the positive integers and for each positive integer $n>1$, let $\mathscr{F}_{n}$ be the filter generated by $N \backslash\{n\}$ and define the convergence structure $q_{n}$ by $q_{n}(1)=\left\{\mathscr{F} \mid \mathscr{F}_{n} \subseteq \mathscr{F}\right.$ or $\left.\mathscr{F}_{n+1} \subseteq \mathscr{F}\right\}$ and $q_{n}$ discrete at all other points of $N$. Then $\vee q_{n}(n>1)$ is a limitierung different from the discrete topology at 1 whereas each $\phi q_{n}$ is indiscrete, $\vee \phi q_{n}=D$ and $\phi$ does not preserve arbitrary joins.

In consequence of Theorem 5.1 we find that each of the lattices of limitierungs (ps., pre.) inherits any property of $C(X)$ preserved 
by lattice homomorphisms. For example, that each of these three lattices is distributive (a well-known result) and that each lattice has pseudo-differences are consequences of Theorem 5.1.

6. Acknowledgment. Most of the results of this paper are contained in the author's dissertation written at the University of Houston under the direction of Professor A. R. Vobach. The author extends his appreciation to the referee for a correction of Theorem 2.5 .

\section{REFERENCES}

1. C. R. Atherton Jr., On the lattice of primitive convergence structures, Can. J. Math., 23 (1971) 392-397.

2. G. Birkhoff, Lattice theory, Amer. Math. Soc. Colloquium Publications 25, Amer. Math. Soc., Providence, R. I. (1967).

3. A. M. Carstens The lattice of pretopologies on an arbitrary set S, Pacific J. Math., 29 (1969) $67-71$.

4. - The lattice of pseudotopologies on S, Portugal Math., 32, (1973), 71-73.

5. G. Choquet, Convergences, Ann. Univ. Grenoble Sect. Sci. Math. Phsy. (N. S.), 23, (1948), 57-112.

6. H. R. Fischer, Limesraume, Math. Ann., 137, (1959), 269-303.

7. B. V. Hearsey and D. C. Kent, Convergence structures, Portugal Math., 31, (1972), 105-118.

8. D. C., Kent, Convergence functions and their related topologies, Fund. Math., 54, (1964) $125-133$.

9. — Convergence quotient maps, Fund. Math., 65, 197-205.

10. D. C. Kent, and C. R. Atherton, The order topology in a bicompactly generated lattice, J. Austral Math. Soc., 8, (1968), 345-349.

11. H. Rasiowa and R. Sikorski, The mathematics of metamathematics, Polish Scientific Publishers, Warsaw, (1968).

12. C. V. Riecke, The lattice of convergence structures, Proc. Univ. Houston Point Set Topology Conf., (1971), 157-163.

13. - Lattice completion of the metrizable topologies, J. Austral Math. Soc., 18, (1974), 447-449.

14. J. Schmidt, Beitrage zur filtertheorie I, Math. Nachr., 7 (1952) 359-378.

15. G. Szasz, Introduction to Lattice Theory, Academic Press, New York, (1963).

16. M. Ward, Structure residuation, Ann. Math., Princeton, II S., 39 (1938) 555-568.

Received July 14, 1976 and in revised form September 28, 1976.

CAMERoN UNIVERSITY

LAWTON, OK 73501 


\section{PACIFIC JOURNAL OF MATHEMATICS}

\section{EDITORS}

RICHARD ARENS (Managing Editor)

University of California

Los Angeles, CA 90024

R. A. BEAUMONT

University of Washington

Seattle, WA 98105

C. C. Moore

University of California

Berkeley, CA 94720

\section{J. DugundJI}

Department of Mathematics

University of Southern California

Los Angeles, CA 90007

R. Finn and J. Milgram

Stanford University

Stanford, CA 94305

\section{ASSOCIATE EDITORS}

\section{E. F. BECKENBACH}

B. H. NEUMANN

F. WoLF

K. YOSHIDA

\section{SUPPORTING INSTITUTIONS}

\author{
UNIVERSITY OF BRITISH COLUMBIA \\ CALIFORNIA INSTITUTE OF TECHNOLOGY \\ UNIVERSITY OF CALIFORNIA \\ MONTANA STATE UNIVERSITY \\ UNIVERSITY OF NEVADA \\ NEW MEXICO STATE UNIVERSITY \\ OREGON STATE UNIVERSITY \\ UNIVERSITY OF OREGON \\ OSAKA UNIVERSITY
}

\author{
UNIVERSITY OF SOUTHERN CALIFORNIA \\ STANFORD UNIVERSITY \\ UNIVERSITY OF HAWAII \\ UNIVERSITY OF TOKYO \\ UNIVERSITY OF UTAH \\ WASHINGTON STATE UNIVERSITY \\ UNIVERSITY OF WASHINGTON \\ AMERICAN MATHEMATICAL SOCIETY
}

The Supporting Institutions listed above contribute to the cost of publication of this Journal, but they are not owners or publishers and have no responsibility for its content or policies.

Mathematical papers intended for publication in the Pacific Jaurnal of Mathematics should be in typed form or offset-reproduced, (not dittoed), double spaced with large margins. Please do not use built up fractions in the text of your manuscript. You may however, use them in the displayed equations. Underline Greek letters in red, German in green, and script in blue. The first paragraph or two must be capable of being used separately as a synopsis of the entire paper. Items of the bibliography should not be cited there unless absolutely necessary, in which case they must be identified by author and Journal, rather than by item number. Manuscripts, in triplicate, may be sent to any one of the editors. Please classify according to the scheme of Math. Reviews, Index to Vol. 39. All other communications should be addressed to the managing editor, or Elaine Barth, University of California, Los Angeles, California, 90024.

The Pacific Journal of Mathematics expects the author's institution to pay page charges, and reserves the right to delay publication for nonpayment of charges in case of financial emergency

100 reprints are provided free for each article, only if page charges have been substantially paid. Additional copies may be obtained at cost in multiples of 50 .

The Pacific Journal of Mathematics is issued monthly as of January 1966. Regular subscription rate: $\$ 7200$ a year (6 Vols., 12 issues). Special rate: $\$ 36.00$ a year to individual members of supporting institutions.

Subscriptions, orders for back numbers, and changes of address should be sent to Pacific Journal of Mathematics, 103 Highland Boulevard, Berkeley, California, 94708.

PUBLISHED BY PACIFIC JOURNAL OF MATHEMATICS, A NON-PROFIT CORPORATION

Printed at Kokusai Bunken Insatsusha (International Academic Printing Co., Ltd.). 8-8, 3-chome, Takadanobaba, Shinjuku-ku, Tokyo 160, Japan.

Copyrit (C) 1975 by Pacific Journal of Mathematics Manufactured and first issued in Japan 


\section{Pacific Journal of Mathematics \\ Vol. 69, No. $2 \quad$ June, 1977}

Carol Alf and Thomas Alfonso O'Connor, Unimodality of the Lévy spectral

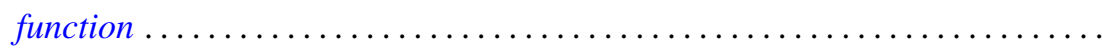

S. J. Bernau and Howard E. Lacey, Bicontractive projections and reordering of

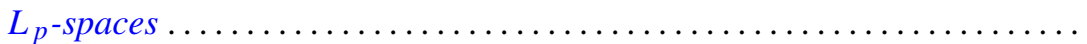

Andrew J. Berner, Products of compact spaces with bi-k and related spaces..... 303

Stephen Richard Bernfeld, The extendability and uniqueness of solutions of ordinary differential equations ...............................

Marilyn Breen, Decompositions for nonclosed planar m-convex sets ..........

Robert F. Brown, Cohomology of homomorphisms of Lie algebras and Lie

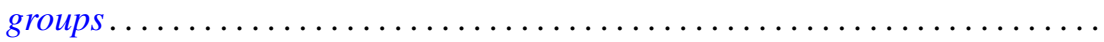

Jack Douglas Bryant and Thomas Francis McCabe, A note on Edelstein's iterative test and spaces of continuous functions ....................

Victor P. Camillo, Modules whose quotients have finite Goldie dimension ....... 333

David Downing and William A. Kirk, A generalization of Caristi's theorem with

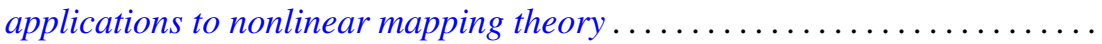

Daniel Reuven Farkas and Robert L. Snider, Noetherian fixed rings ...........

Alessandro Figà-Talamanca, Positive definite functions which vanish at

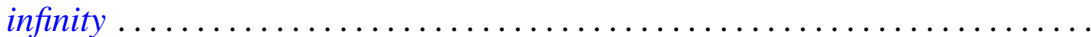

Josip Globevnik, The range of analytic extensions .................. 365

André Goldman, Mesures cylindriques, mesures vectorielles et questions de

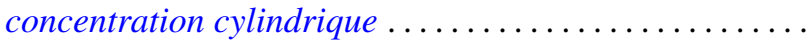

Richard Grassl, Multisectioned partitions of integers..........

Haruo Kitahara and Shinsuke Yorozu, A formula for the normal part of the

Laplace-Beltrami operator on the foliated manifold .... .

Marvin J. Kohn, Summability $R_{r}$ for double series .........

Charles Philip Lanski, Lie ideals and derivations in rings with involution ..

Solomon Leader, A topological characterization of Banach contractions . .

Daniel Francis Xavier O’Reilly, Cobordism classes of fiber bundles . .

James William Pendergrass, The Schur subgroup of the Brauer group . .

Howard Lewis Penn, Inner-outer factorization of functions whose Fourier series

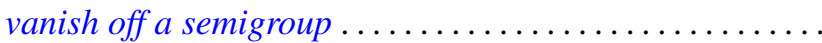

501

William T. Reid, Some results on the Floquet theory for disconjugate definite

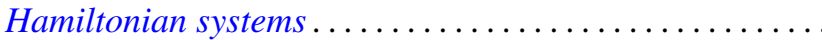

Caroll Vernon Riecke, Complementation in the lattice of convergence

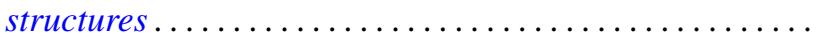

Louis Halle Rowen, Classes of rings torsion-free over their centers ......... 527

Manda Butchi Suryanarayana, A Sobolev space and a Darboux problem ....... 535

Charles Thomas Tucker, II, Riesz homomorphisms and positive linear maps.... 551

William W. Williams, Semigroups with identity on Peano continua ........... 557

Yukinobu Yajima, On spaces which have a closure-preserving cover by finite 